\title{
Correction to: Metabolomic Fingerprinting of Blood Samples by Direct Infusion Mass Spectrometry: Application in Alzheimer's Disease Research
}

Raúl González-Domínguez ${ }^{1}$ [

Published online: 9 October 2017

(c) The Nonferrous Metals Society of China and Springer Nature Singapore Pte Ltd. 2017

Correction to: J. Anal. Test. (2017) 1:17

DOI 10.1007/s41664-017-0018-4

Unfortunately, the following article was published in the previous issue by mistake. This article is a part of this Special Issue.

The online version of the original article can be found under doi:10.1007/s41664-017-0018-4.

Raúl González-Domínguez

raul.gonzalez@dqcm.uhu.es

1 Department of Chemistry, Faculty of Experimental Sciences,

University of Huelva, 21007 Huelva, Spain 


\title{
Metabolomic Fingerprinting of Blood Samples by Direct Infusion Mass Spectrometry: Application in Alzheimer's Disease Research
}

\author{
Raúl González-Domínguez ${ }^{1}$ (D)
}

Received: 11 March 2017/Accepted: 19 June 2017/Published online: 27 June 2017

(C) The Nonferrous Metals Society of China and Springer Nature Singapore Pte Ltd. 2017

\begin{abstract}
Metabolomics is largely employed in numerous biomedical research fields, such as the study of the underlying pathology of diseases, discovery of diagnostic biomarkers, or drug development. Nowadays, the main challenge is to obtain comprehensive and unbiased metabolomic profiles due to the huge complexity, heterogeneity, and dynamism of the metabolome. To this end, mass spectrometry represents a very interesting analytical platform, since the complexity of metabolome may be overcome through the use of different orthogonal separation techniques, including liquid chromatography, gas chromatography, and capillary electrophoresis. Alternatively, direct mass spectrometry analysis has been postulated as a complementary choice to hyphenated approaches. This technique exhibits several advantages such as the ability for high-throughput screening, fast analysis, and wide metabolomic coverage, since there is not exclusion of compounds due to the separation device. The present work explores the utility of metabolomics based on direct infusion mass spectrometry for analyzing blood samples. The most important analytical concerns to be considered are discussed, including sample handling, comprehensive fingerprinting, as well as subsequent identification of metabolites, and global characterization of metabolomic profiles. To conclude, a brief review on the application of these metabolomic platforms in Alzheimer's disease research is also provided.
\end{abstract}

Raúl González-Domínguez

raul.gonzalez@dqcm.uhu.es

1 Department of Chemistry, Faculty of Experimental Sciences, University of Huelva, 21007 Huelva, Spain
Keywords Metabolomics · Direct infusion mass spectrometry · Alzheimer's disease $\cdot$ Blood

\section{Introduction}

Two complementary analytical platforms have predominantly been employed in metabolomic research: nuclear magnetic resonance (NMR) and mass spectrometry (MS). The potential of NMR for high-throughput analysis, along with its non-destructive character, has traditionally made this technique a very useful workhorse in metabolomics [1]. However, mass spectrometry has gained great importance in the last years due to its increased sensitivity and specificity, as well as because of its higher suitability for studying complex samples [2]. Furthermore, it is noteworthy that MS can be used to perform both qualitative and quantitative analyses, thus allowing classification of samples by means of metabolomic fingerprinting and subsequent identification of altered metabolites via fragmentation experiments. Mass spectrometry is usually coupled to various separation techniques, such as liquid chromatography (LC), gas chromatography (GC), or capillary electrophoresis (CE), in order to resolve isobaric interferences and to reduce ion suppression [3, 4]. Nevertheless, these hyphenated approaches considerably increase the total analysis time and, consequently, the inter-sample variability, which hinders obtaining valid biological information from metabolomic data. In this context, direct infusion mass spectrometry (DIMS) has emerged as a very interesting alternative with the aim to overcome these drawbacks. DIMS-based metabolomics exhibits multiple advantages, including high-throughput screening capability because of the reduced analysis time, as well as comprehensive metabolomic coverage, thanks to the lack of a 
previous separation step, which prevents the inherent analytical bias introduced by these selective retention mechanisms and enables the simultaneous determination of a broad range of metabolites with very different physicochemical characteristics [5]. Thus, direct MS analysis has been postulated as a suitable tool to perform a preliminary metabolomic exploration of complex samples prior to the application of orthogonal hyphenated platforms [6].

Metabolomics based on direct infusion MS has demonstrated a great potential in numerous areas, such as disease phenotyping, nutritional research, food characterization and authentication, and environmental monitoring, as recently reviewed by several authors [5, 7]. Nevertheless, it should be noted that special care must be taken during the optimization of these analytical tools in order to maximize metabolomic coverage, particularly with regard to sample extraction and non-targeted MS fingerprinting. The selection of a proper extraction protocol is crucial in shotgun metabolomics in order to recover as many metabolites as possible from the sample. In the case of biofluids, sample treatment is usually performed by simple protein precipitation with organic solvents, while the study of tissues requires a previous step of homogenization and cellular lysis [8]. However, these procedures usually fail to extract lipophilic components that may remain adsorbed into the protein precipitates. On the other hand, another important point to be considered is the ionization conditions applied to acquire metabolomic profiles. Because of the high complexity of metabolome, different subsets of metabolites can be detected depending on the ionization polarity employed, although the positive ion mode usually provides much higher sensitivity. Moreover, the possibility of combining complementary ionization sources, such as electrospray ionization (ESI), atmospheric pressure chemical ionization (APCI), or atmospheric pressure photoionization (APPI), opens the range of measurable analytes [9]. Therefore, the combination of exhaustive sample extraction procedures with non-biased MS analysis, usually based on the complementary use of both positive and negative ion modes as well as different ionization sources, could provide a very powerful analytical approach for comprehensive metabolomic fingerprinting.

The application of these high-throughput metabolomic approaches has emerged in recent years as a very powerful strategy in biomedical research to investigate the pathogenesis of diseases and to discover candidate biomarkers for diagnosis. Particularly, these tools are very useful for studying complex and heterogeneous disorders, such as Alzheimer's disease (AD). The exact etiology of this neurodegenerative disorder is still unclear, but there is growing evidence about the involvement of multiple pathological hallmarks, including abnormal metabolism of the amyloid precursor protein, tau hyperphosphorylation, oxidative stress, mitochondrial dysfunction, abnormal metal homeostasis, and many others [10-12]. Thus, there is great interest in identifying the totality of functionally interrelated pathological changes associated with $\mathrm{AD}$ development and progression, for which non-targeted metabolomic analysis has previously demonstrated a huge potential, as recently reviewed $[6,13]$. In this context, it is noteworthy that cerebrospinal fluid (CSF) is the most commonly employed biological matrix for diagnosing neurological disorders because its composition directly reflects brain neurochemical alterations. However, the use of peripheral samples such as blood serum or plasma is a very interesting alternative with the aim to identify simpler and cheaper biomarkers, easily translatable to the clinical practice [14].

The present review article explores the potential of direct MS analysis for serum/plasma metabolomics, with a particular emphasis on critical issues such as sample handling, comprehensive metabolomic analysis, as well as subsequent identification of metabolites, and global characterization of metabolomic fingerprints. To this end, this work is focused on previously published papers dealing with the application of these metabolomic platforms for the elucidation of metabolic disturbances underlying Alzheimer's disease (AD) and the discovery of candidate biomarkers for diagnosis.

\section{Direct Infusion Mass Spectrometry for Blood Metabolomics}

Blood plasma and serum are the commonly employed biological samples in biomedical research due to their ability to reflect physiological responses to external stimuli, as well as to their easy accessibility, they since can be drawn in any physician's office as part of routine clinical analyses, unlike tissue (e.g., biopsies) or cerebrospinal fluid that must be obtained using invasive, and usually more expensive, sampling procedures. These peripheral samples contain high concentrations of several macromolecules, principally proteins and peptides, which must be removed prior to metabolomic analysis since they can provoke important interferences. In this sense, it should be noted that comprehensive metabolite fingerprinting requires simple and universal sample preparation protocols in order to avoid analyte losses. For this reason, deproteinization and metabolite extraction using organic solvents (e.g., methanol, acetonitrile, ethanol) has been established as the most common procedure when analyzing blood samples [8], which allows obtaining holistic metabolomic profiles in a wide range of physicochemical properties. However, some metabolites in serum and plasma can be bound to proteins, so exhaustive extraction processes are needed to 
degrade metabolite-protein complexes. This is the case of highly lipophilic lipids, such as triglycerides, which may remain adsorbed into the protein fraction during the precipitation step due to their low solubility in aqueous solutions. To solve this problem, some authors have proposed the application of traditional methods for the extraction of lipids, such as the Bligh-Dyer protocol, with a great potential in lipidomic analysis [15]. However, the high lipid content of blood samples may cause ion suppression and mask the detection of other low-molecular weight metabolites, so that the use of sequential extraction procedures has become a very interesting alternative. In this sense, González-Domínguez et al. optimized a reproducible and sensitive metabolomic approach based on a two-step sample treatment and DIMS analysis for serum fingerprinting [16]. First, conventional protein precipitation was applied to obtain methanolic extracts containing polar metabolites, and then protein precipitates were again extracted with non-polar solvents to recover lipophilic compounds adsorbed into the protein fraction. On the other hand, the dilution factor is another critical issue to be considered during the optimization of the sample preparation methodology since it plays a pivotal role in the attenuation of matrix effects. Therefore, it has been demonstrated that the relationship between signal intensity and metabolite concentration is generally linear at higher sample dilutions, given that the high number of ions in concentrated samples may reduce sensitivity due to ion suppression, while at higher dilutions the beneficial effect of diminished ion suppression is lost [17].

After the extraction of metabolites, direct MS fingerprinting can be accomplished by means of different instrumental configurations [5]. Direct infusion mass spectrometry (DIMS) uses a syringe pump to constantly introduce sample extracts into the mass spectrometer, while flow injection mass spectrometry (FI-MS) is based on the infusion of samples as a plug into a stream of solvent delivered by a LC pump. With regard to the ionization source, electrospray ionization (ESI) is the most common choice due to its high sensitivity and versatility, which enables the simultaneous detection of compounds with very different polarities and molecular weights. Complementarily, atmospheric pressure chemical ionization and atmospheric pressure photoionization sources are more efficient variants for ionizing less polar compounds, with lower susceptibility to ion suppression effects than ESI. Thus, the use of different ionization sources, operating in both positive and negative ion modes, is recommended in order to maximize metabolome coverage.

The application of these high-throughput DIMS-based metabolomic approaches provides characteristic metabolic snapshots comprising numerous circulating metabolites. For instance, the analytical platform recently developed by
González-Domínguez allows studying a large number of metabolites by means of the integration of four complementary metabolic fingerprints obtained after the analysis of polar and lipophilic extracts using DI-ESI-MS in both positive and negative modes of ionization (Fig. 1) [16]. Metabolomic profiles acquired in positive ion mode (ESI+) exhibit numerous signals in a wide range of molecular weights, thus evidencing the great complexity of the blood metabolome. Polar extracts contain numerous low-molecular weight (LMW) hydrophilic metabolites, resulting in complex spectra in the $\mathrm{m} / \mathrm{z}$ range $50-400$ (Fig. 1a). On the other hand, clusters of high-molecular mass signals (i.e., above $\mathrm{m} / \mathrm{z}$ 500) were predominant in lipophilic extracts (Fig. 1b), which could be allocated to various classes of lipids. Alternatively, the analysis of these sample extracts in negative ion mode (ESI-) yielded metabolomic profiles with a considerably lower number of molecular features. The most intense signals were observed in the LMW region of polar extracts (i.e. $m / z<400$, Fig. 1c), although some high-molecular mass metabolites could also be detected, principally by analyzing lipophilic extracts (Fig. 1d). Therefore, it could be concluded that the combination of sequential extraction procedures and subsequent analysis by means of complementary ionization mechanisms allows obtaining very informative metabolomic profiles. Particularly, it is worth noting the usefulness of using a second extraction step to recover lowpolarity metabolites after protein removal (Fig. 1c, d), which provide very valuable metabolic information due to the pivotal role of lipids in biological systems. Furthermore, the integration of data acquired in positive and negative ionization modes enables the detection of metabolites with very different physicochemical properties (e.g., acidity and polarity), thus helping to get deeper insights into the complex human metabolome.

\section{Characterization of DIMS-based Metabolomic Fingerprints}

The main objective in metabolomic research is the identification of discriminant metabolites among the study groups with the aim to elucidate the biological mechanisms underlying a pathological status or external stimuli, and to discover potential biomarkers of diagnosis or exposure. In this context, the Metabolomics Standards Initiative (MSI) establishes four levels of metabolite identification according to the following guidelines [18]. Definitive annotation (level I) requires the analysis of authentic standards under identical instrumental conditions employed in metabolomic experiments in order to validate the identity of these compounds on the basis of a minimum of two orthogonal and independent data (e.g., accurate mass, tandem MS, 

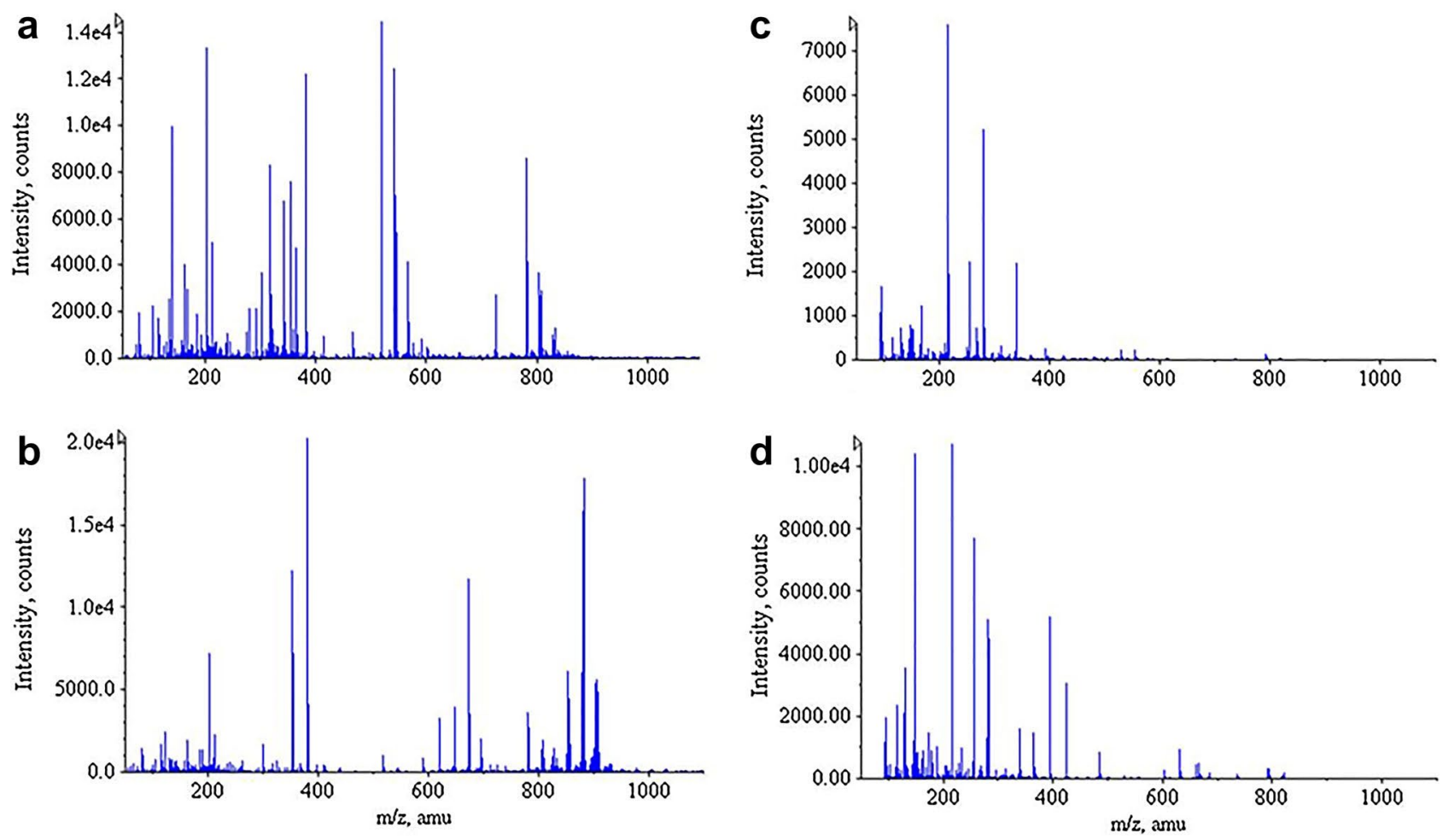

Fig. 1 Metabolomic fingerprints obtained by direct infusion electrospray mass spectrometry (DI-ESI-MS) analysis of serum samples: a DI-ESI(+)-MS analysis of aqueous extracts; b DI-ESI(+)-MS analysis of lipophilic extracts; $\mathbf{c}$ DI-ESI(-)-MS analysis of aqueous

retention time). However, this is not always possible due to the lack of commercial standards for multiple metabolites, as well as because of the presence of a myriad of isomers in biological samples, especially for lipid compounds. Another alternative is the querying of experimental data against metabolomic databases, without chemical reference standards (level II). This procedure has largely been employed in numerous previously published metabolomic studies to perform a putative annotation of metabolites, but a great number of discriminant signals usually remain unidentified due to the limitations of existing libraries, most of them still under development [19]. Finally, when a particular metabolite cannot be allocated following the two first levels of identification, it can be putatively characterized according to the chemical class to which it belongs (level III), or it can be reported as "unknown" classifier (level IV). Therefore, it is noteworthy that the annotation step is still a very important bottleneck in metabolomics. For this reason, the knowledge of tandem MS spectra of the most common metabolite classes can be of great help with the aim to facilitate the identification of discriminant compounds. In this section, a detailed discussion of metabolite fragmentation patterns is provided in order to accomplish a global characterization of metabolomic fingerprints obtained by applying the previously optimized

extracts; and d DI-ESI(-)-MS analysis of lipophilic extracts (Reprinted from González-Domínguez et al. [11] with permission from Springer)

DI-ESI-MS methodology [16] and thus demonstrate the potential of this platform for comprehensive serum/plasma metabolomics, as summarized in Fig. 2.

\subsection{Low-Molecular Weight Metabolites}

This category encompasses a very large number of different low-molecular weight metabolites (LMWM, $\mathrm{m} / \mathrm{z}$ range 50-300), with a great compositional diversity. The most important classes of endogenous LMW metabolites include amino acids, carbohydrates, organic acids, nucleotide derivatives (e.g., purines and pyrimidines), alcohols, and amines, among many others [20]. However, the human metabolome is also composed of other compounds from exogenous origin (e.g., drugs, pollutants) as well as microbiota-derived metabolites, thus significantly increasing the complexity of resulting metabolomic profiles. This structural and physicochemical heterogeneity considerably hinders the annotation of metabolites, which usually requires the combination of multiple MS data [21]. The identification of metabolomic features of interest generally begins with the calculation of the empirical formula on the basis of the accurate molecular mass, for which several chemical rules can be applied such as the nitrogen rule, the rings and double bonds formula, and the octet rule. The 

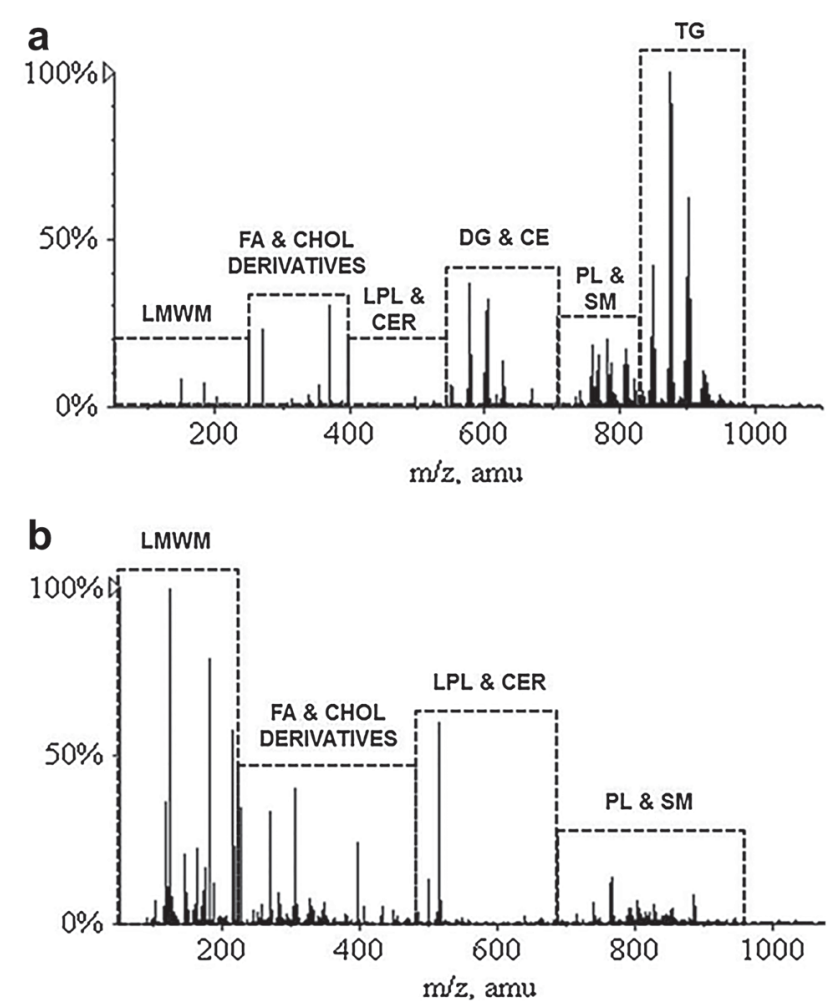

Fig. 2 Global characterization of metabolomic fingerprints obtained by DI-ESI(+)-MS (a) and DI-ESI(-)MS (b) analysis of serum samples

isotopic pattern also provides very useful information with the aim to deduce the total number of carbon atoms and the presence of heteroelements in the molecule (e.g., halogens, sulfur). Then, tandem MS data can be used for searching functional groups and to elucidate the metabolite structure, but the most common practice is the comparison of obtained fragmentation spectra with those available in mass spectral libraries, since de novo identification of metabolites is a very time-consuming procedure. However, it should be noted that only $5-10 \%$ of metabolites reported in the most important metabolomic databases, such as the Human Metabolome DataBase (HMDB) and METLIN, have annotated spectral data. Anyway, numerous LMW metabolites can be identified in human serum/plasma samples by DIMS analysis in both positive and negative modes of ionization, as shown by the great number of signals observed in metabolomic fingerprints (Fig. 2).

\subsection{Fatty Acids and Derivatives}

Free fatty acids (FFA), also named non-esterified fatty acids (NEFA), are present at relatively elevated concentrations in blood circulation, being long-chain fatty acids (i.e., 16-20 carbon atoms) the most abundant species. These lipids can be detected in the negative ionization mode in the form of deprotonated adducts, yielding very intense signals in the $\mathrm{m} / \mathrm{z}$ range $150-350$ (Fig. 2b). However, it should be noted that in-source fragmentation of derived lipids, principally highly concentrated phospholipids, may also have an important contribution to these signals, so that findings obtained by DIMS fingerprinting with regard to FFA homeostasis should be subsequently validated using complementary analytical approaches, such as gas chromatography coupled to mass spectrometry.

The oxidation of arachidonic acid, and to a lesser extent other polyunsaturated acids, by the action of cyclooxygenases, lipoxygenases, various cytochromes $\mathrm{P} 450$, as well as other non-enzymatic pathways, generates a myriad of signaling molecules known as eicosanoids. There are numerous classes of these hydroxylated oxidation products, including prostaglandins, leukotrienes, lipoxins, tromboxanes, hydroxyeicosatetraenoic acids (HETE), and hydroxyeicosapentaenoic acids (HEPE) among others, but the similarities in their structures and chemical characteristics allow using fragmentation experiments to allocate eicosanoid signals in DIMS metabolomic profiles. Eicosanoids can be readily detected as carboxylate anions in the negative mode of analysis (i.e., $[\mathrm{M}-\mathrm{H}]^{-}$), but protonated molecular ions produced under electrospray positive ionization are not stable and usually underwent the loss of one or two molecules of water (i.e., $\left[\mathrm{M}+\mathrm{H}-\mathrm{H}_{2} \mathrm{O}\right]^{+},[\mathrm{M}+\mathrm{H}-$ $\left.2 \mathrm{H}_{2} \mathrm{O}\right]^{+}$). These signals can be found in a narrow $\mathrm{m} /$ $z$ window $(\mathrm{m} / \mathrm{z}, 300-400 \mathrm{Da})$ and show characteristic tandem mass spectra dominated by the presence of fragments derived from the loss of the carboxylic moiety [22]. Anyway, it is worth noting that isomeric forms of these eicosanoids cannot be distinguished by DI-ESI-MS fingerprinting, which usually requires the application of LC-based methodologies.

Acylcarnitines are another class of biologically relevant metabolites derived from fatty acids that can be easily detected by DIMS-based analysis in blood samples and, especially, in tissues. The ionization of these compounds predominantly yields protonated ions $[\mathrm{M}+\mathrm{H}]^{+}$with molecular masses ranging from 200 to 500 Da (Fig. 2a). The fragmentation pattern of acylcarnitines is characterized by two signals at $m / z 60$ and $[\mathrm{M}+\mathrm{H}-59]^{+}$, derived from the loss of the trimethylamine moiety, as well as by a common fragment at $\mathrm{m} / \mathrm{z} 85$, corresponding to a rearrangement of the butyric acid chain to generate the cation $\left[\mathrm{CH}_{2}-\right.$ $\left.\mathrm{CH}=\mathrm{CH}-\mathrm{CO}_{2} \mathrm{H}\right]^{+}[23]$.

To conclude, several primary fatty acid amides (PFAAs) can also be found in the organism. Similarly to acylcarnitines, the most common PFAAs are detected as $[\mathrm{M}+\mathrm{H}]^{+}$adducts in DI-ESI(+)-MS fingerprints in the $m /$ $z$ range $200-400$. The collision-induced product ion mass spectra of these lipids are dominated by an acyl chain fragment observed at $m / z \quad 57$ [24]. Moreover, 
monounsaturated PFAAs present a distinct fragmentation pattern characterized by neutral losses of 17 and $35 \mathrm{Da}$, corresponding to fragments derived from the elimination of ammonia (i.e., $\left[\mathrm{M}+\mathrm{H}-\mathrm{NH}_{3}\right]^{+}$) and both ammonia and water (i.e., $\left[\mathrm{M}+\mathrm{H}-\mathrm{NH}_{3}-\mathrm{H}_{2} \mathrm{O}\right]^{+}$) from the carboxamide head group.

\subsection{Glycerolipids}

Glycerolipids constitute a class of abundant neutral lipids, composed by mono-, di-, and tri-esterified glycerol with different fatty acids. These lipids can only be readily ionized in positive mode, and requires the use of lipophilic solvents to be extracted from biological samples. For this reason, glycerolipids are usually not detected in most of the previously published metabolomic studies based on simple protein precipitation with organic solvents. The ionization of mono- and diglycerides can be accomplished via the formation of dehydrated molecular ions $\left[\mathrm{M}+\mathrm{H}-\mathrm{H}_{2} \mathrm{O}\right]^{+}$, thanks to the presence of free hydroxyl groups. However, the most common ionization mechanism for these neutral lipids is the attachment of charged ions, such as sodium or, especially, ammonium [25]. Therefore, various peak clusters can be observed in DI-ESI(+)-MS metabolomic fingerprints when lipophilic extracts are analyzed, located around $\mathrm{m} / \mathrm{z}, 300-400$ for monoglycerides, $\mathrm{m} / z$ 550-700 for diglycerides, and $m / z$ 800-1000 for triglycerides. Interestingly, glycerolipids show a very informative fragmentation pattern characterized by the presence of four types of ions, namely A, B, C, and D ions, which are generated through the release of fatty acids from the glycerol backbone [26]. These ions present characteristic $\mathrm{m} / \mathrm{z}$ values depending on the fatty acid contained in the glycerolipid structure as shown in Table 1, which facilitates the annotation of these compounds and other related lipids, as discussed in the next section.

Table 1 Characteristic $\mathrm{m} / \mathrm{z}$ values of B, C, and D fragment ions for the most common fatty acids

\begin{tabular}{lllll}
\hline Fatty acid & Molecular mass & Ion B & Ion C & Ion D \\
\hline Myristic acid & 228.209 & 285.24 & 211.21 & 193.19 \\
Palmitoleic acid & 254.225 & 311.26 & 237.22 & 219.21 \\
Palmitic acid & 256.24 & 313.27 & 239.24 & 221.23 \\
Linolenic acid & 278.225 & 335.26 & 261.22 & 243.21 \\
Linoleic acid & 280.240 & 337.27 & 263.24 & 245.23 \\
Oleic acid & 282.256 & 339.29 & 265.25 & 247.24 \\
Stearic acid & 284.272 & 341.31 & 267.27 & 249.26 \\
Eicosapentaenoic acid & 302.225 & 359.26 & 285.22 & 267.21 \\
Arachidonic acid & 304.240 & 361.27 & 287.24 & 269.23 \\
Docosahexaenoic acid & 328.240 & 385.27 & 311.24 & 293.23 \\
\hline
\end{tabular}

\subsection{Phospholipids and Sphingolipids}

Phospholipids and sphingolipids are the major constituents of cellular membranes and serve as important reservoir of fatty acids and related compounds such as eicosanoids. There are various classes of these lipids differentially distributed across the different organs and biological compartments of the organism, but all of them show similar fragmentation patterns that enable their annotation in a simple manner.

Phospholipids (PL) consist of a glycerol backbone esterified with two fatty acids and a characteristic phosphate ester group in the $s n-3$ position. Depending on the chemical nature of the polar group linked to the phosphate moiety, six major classes of phospholipids can be formed, including phosphatidylcholines (PC), phosphatidylethanolamines (PE), phosphatidylinositols (PI), phosphatidylserines (PS), phosphatidylglycerols (PG), and phosphatidic acids (PA). Another possibility is the presence of an alkyl or, especially, a vinyl ether group in the $s n-1$ position, leading to the so-called plasmalogens, usually containing choline and ethanolamine head groups (i.e., plasmenylcholines and plasmenylethanolamines). Furthermore, high concentrations of lysophospholipids (LPL) can also be found in serum and plasma samples, generated by hydrolysis of one fatty acid substituent from phospholipids. Because of the high ionization efficiency of these lipids under ESI-MS, DIMS fingerprints contain numerous phospholipid-derived signals in the high-molecular mass region of spectra acquired in both positive and negative ion modes ( $\mathrm{m} / \mathrm{z}, 450-600$ for LPLs, $\mathrm{m} / \mathrm{z}, 700-900$ for PLs), as shown in Fig. 2. Choline-containing phospholipids are usually detected as protonated $\left([\mathrm{M}+\mathrm{H}]^{+}\right)$, sodiated $\left([\mathrm{M}+\mathrm{Na}]^{+}\right)$, and potassiated $\left([\mathrm{M}+\mathrm{K}]^{+}\right)$ions in positive mode, while in negative polarity these lipids generate demethylated ions $\left(\left[\mathrm{M}-\mathrm{CH}_{3}\right]^{-}\right)$, chloride adducts $\left([\mathrm{M}+\mathrm{Cl}]^{-}\right)$, or formate adducts $\left([\mathrm{M}+\mathrm{FA}]^{-}\right)$. The most abundant ions for ethanolamine, serine, and glycerol species are $[\mathrm{M}+\mathrm{H}]^{+}$and $[\mathrm{M}-\mathrm{H}]^{-}$, in positive and negative modes, respectively. On the other hand, phosphatidylinositols and phosphatidic acids are mainly detected in negative ion mode in the form of deprotonated ions. Phosphatidylcholines and lysophosphatidylcholines present characteristic fragments in positive ionization mode at $\mathrm{m} / \mathrm{z}$ 184.07, 104.10, and 86.09, and two typical neutral losses due to the breakage of trimethylamine $(\mathrm{m} / \mathrm{z} 59)$ and phosphocholine head group $(\mathrm{m} / \mathrm{z} 183,205$, or 221 , if the counter ion is proton, sodium, or potassium, respectively). By contrast, product ion spectra of ethanolamines, serines, and glycerols are dominated by $[\mathrm{M}+\mathrm{H}-141]^{+},[\mathrm{M}+\mathrm{H}-185]^{+}$, and $[\mathrm{M}+\mathrm{H}-172]^{+}$, respectively, arising from the elimination of the phosphoethanolamine, phosphoserine, and phosphoglycerol moieties. Alternatively, these distinctive signals 
are found in negative ion mode at $\mathrm{m} / \mathrm{z} 153.00,168.04$, 196.04, 241.02, 171.01, and [M-H-87] ${ }^{-}$, for phosphatidic acid-, choline-, ethanolamine-, inositol-, glycerol-, and serine-derived lipids, respectively [27]. Furthermore, it is noteworthy that MS/MS breakage of ester linkages between fatty acids and the glycerol backbone enables the identification of individual species of phospholipids by means of the interpretation of ions B, C, and D generated in positive ion mode (Table 1), or by the presence of daughter ions related to the corresponding carboxylate anions released under ESI(-)-MS fragmentation [28, 29].

On the other hand, the structure of sphingolipids (SL) is based on a long-chain base of the sphingosine family with an amide-linked fatty acid. These SL precursors are called ceramides (CER), and they can be converted into sphingomyelins (SM) via the esterification of the ending hydroxyl group with a phosphocholine moiety. These lipids show a similar distribution in DIMS profiles to that mentioned for phospholipids, with ceramides located in the $\mathrm{m} /$ $z$ range 500-600 and sphingomyelins clustered around 700-800 Da (Fig. 2). After tandem MS fragmentation, sphingolipids yield typical product ions at $\mathrm{m} / \mathrm{z} 264.27$ and 282.28 due to the fragmentation in the sphingosine moiety, while the cleavage of the phosphocholine head group from sphingomyelins generates characteristic fragments at $\mathrm{m} /$ z 184.07 and 168.04 in positive and negative ion modes, respectively [30].

\subsection{Cholesterol and Derivatives}

Various cholesterol derivatives can also be fingerprinted by DI-ESI-MS, principally using the positive ionization mode. Free cholesterol is usually detected in lipophilic extracts as a very intense signal at $m / z, 369.35$, allocable to the $[\mathrm{M}+\mathrm{H}-$ $\left.\mathrm{H}_{2} \mathrm{O}\right]^{+}$molecular ion; but the formation of $\left[\mathrm{M}+\mathrm{NH}_{4}\right]^{+}$ adducts $(\mathrm{m} / \mathrm{z}, 404.39)$ is possible when ammonium salts are added to sample extracts, as described for glycerolipids. Similarly, cholesteryl esters (CE) are also exclusively detected by ESI(+)-MS analysis of lipophilic extracts in the form of adducts with common cations, such as ammonium and sodium, and they can be readily annotated, thanks to the abundant fragment ion corresponding to the cholesterol moiety $(\mathrm{m} / \mathrm{z}, 369.35)$, which is originated after the hydrolysis of the ester bond upon collision-induced dissociation [31]. Finally, multiple bile acids (BA) are also easily observed in metabolomic fingerprints shown in Fig. 2, ranging from 350 to $500 \mathrm{Da}$. In positive ion mode, MS signals can be allocated to $[\mathrm{M}+\mathrm{H}]^{+},\left[\mathrm{M}+\mathrm{H}-\mathrm{H}_{2} \mathrm{O}\right]^{+}$, and $\left[\mathrm{M}+\mathrm{NH}_{4}\right]^{+}$adducts, while the use of negative ionization predominantly yields $[\mathrm{M}-\mathrm{H}]^{-}$and $\left[\mathrm{M}-\mathrm{H}-\mathrm{H}_{2} \mathrm{O}\right]^{-}$ ions. Tandem mass spectra of these bile acids are dominated by dehydrated ions with the formula $\left[\mathrm{M}+\mathrm{H}-\mathrm{nH}_{2} \mathrm{O}\right]^{+}$, where $n$ represents the number of hydroxyl groups on the steroid skeleton. On the other hand, fragmentation under ESI(-)-MS usually involves the sequential elimination of $\mathrm{H}_{2} \mathrm{O}, \mathrm{CO}_{2}$, and $\mathrm{H}_{2} \mathrm{CO}_{2}$ from the parent structure, and the production of typical fragments at $\mathrm{m} / \mathrm{z} 74$ for glycine conjugates as well as at $\mathrm{m} / \mathrm{z}, 124$ and 80 for taurine conjugated bile acids [32].

\section{Application of DIMS Metabolomic Fingerprinting in Alzheimer's Disease Research}

Alzheimer's disease (AD) is characterized by neuropathological alterations originated by the deposition of amyloid $\beta$ plaques and the formation of intraneuronal neurofibrillary tangles of hyperphosphorylated tau $\tau$ protein. However, there is growing evidence that other numerous cellular processes can also be involved in the pathogenesis of this disorder, including oxidative stress, mitochondrial dysfunction, abnormal metal homeostasis, and many others [10-12]. For this reason, the application of holistic metabolomic approaches has demonstrated a great potential for the investigation of this multifactorial disorder. Particularly, the use of direct MS platforms has previously been described in a few research articles with the aim to elucidate pathological hallmarks associated with $\mathrm{AD}$ development and progression, in both human cohorts and transgenic animal models, as briefly discussed in this section. The methodology optimized by González-Domínguez et al., based on a two-step extraction procedure and subsequent DI-ESI-MS fingerprinting of serum samples, was successfully employed to identify discriminant metabolites between $\mathrm{AD}$ patients and healthy control subjects [16, 33], as well as between various transgenic mice lines and wildtype littermates [34, 35]. These studies revealed significant disturbances in the homeostasis of various lipid classes (e.g., phospholipids, fatty acids, glycerolipids), energy-related metabolites, neurotransmitters, and other metabolites, thus evidencing the utility of simple and easily available serum samples in clinical research. Furthermore, these findings were then validated using orthogonal hyphenated approaches, such as UHPLC-MS [36, 37], GC-MS [37, 38], and CE-MS [39], demonstrating the reliability of direct MS-based metabolomics. This DIMS platform was latterly adapted for the analysis of other biological matrices, such as urine [40], brain [41], peripheral organs (i.e., liver, kidney, thymus, spleen) [42], as well as muscle, hepatopancreas, and antennal gland [43], providing very valuable results to accomplish a preliminary metabolomic screening prior to the application of other complementary techniques. Lin et al. also described the application of DIMS-based metabolomics to investigate the characteristic neurochemical profile of the CRND8 transgenic mice. They found increased hippocampal production of 
neuroinflammatory eicosanoids [44], as well as profound metabolic perturbations related to abnormal metabolism of purines and fatty acids in the cerebellum [45]. Furthermore, it is also worth noting the utility of combining complementary ionization sources in order to maximize metabolomic coverage, as recently demonstrated by applying FIAPPI-MS fingerprinting on serum samples from AD patients [46] and APP $\times$ PS1 mice [34]. The APPI source can be used for ionizing both polar and non-polar compounds through complementary ionization mechanisms, thus complementing ionization capabilities of ESI and opening the range of measurable metabolites. Alternatively, some authors have also demonstrated the utility of direct MS analysis to accomplish a comprehensive lipidomic characterization of multiple biological samples from both $\mathrm{AD}$ patients and transgenic animal models. As previously described, González-Domínguez et al. employed a modification of the Bligh-Dyer extraction protocol to investigate the serum lipidome associated with $\mathrm{AD}$ pathogenesis [15]. Therefore, a large number of neutral lipids could be fingerprinted, including diglycerides, triglycerides, and cholesterol derivatives, which are not easily detectable by conventional metabolomic approaches. More recently, a targeted lipidomic study also showed significant perturbations in circulating levels of plasmalogens and diglycerides [47], thus corroborating the pivotal role of lipids in $\mathrm{AD}$ development. To conclude, it should also be noted the works conducted by Han et al. by applying multidimensional mass spectrometry-based lipidomics (MDMS-SL) in plasma and brain samples, which revealed significant deficiencies in levels of plasmalogens [48], sulfatides [49-51], and sphingomyelins [52], as well as increased ceramides [49, 52], in line with previous studies.

Therefore, it can be concluded that high-throughput DIMS-based metabolomics presents a great potential for studying complex disorders such as Alzheimer's disease, and it could be hypothesized that it will play a crucial role in the near future of biomedical research for the elucidation of pathological mechanisms underlying diseases and the discovery of candidate biomarkers for diagnosis.

\section{References}

1. Emwas AHM, Salek RM, Griffin JL, Merzaban J. NMR-based metabolomics in human disease diagnosis: applications, limitations, and recommendations. Metabolomics. 2013;9:1048-72.

2. Dettmer K, Aronov PA, Hammock BD. Mass spectrometry-based metabolomics. Mass Spectrom Rev. 2007;26:51-78.

3. Kuehnbaum NL, Britz-McKibbin P. New advances in separation science for metabolomics: resolving chemical diversity in a postgenomic era. Chem Rev. 2013;113:2437-68.

4. Barbas C, Moraes EP, Villaseñor A. Capillary electrophoresis as a metabolomics tool for non-targeted fingerprinting of biological samples. J Pharm Biomed Anal. 2011;55:823-31.
5. González-Domínguez R, Sayago A, Fernández-Recamales A. Direct infusion mass spectrometry for metabolomic phenotyping of diseases. Bioanalysis. 2017;9:131-48.

6. González-Domínguez R, Sayago A, Fernández-Recamales A. Metabolomics in Alzheimer's disease: the need of complementary analytical platforms for the identification of biomarkers to unravel the underlying pathology. J Chromatogr B. 2017 (in press).

7. Draper J, Lloyd AJ, Goodacre R, Beckmann M. Flow infusion electrospray ionisation mass spectrometry for high throughput, non-targeted metabolite fingerprinting: a review. Metabolomics. 2013;9:S4-29.

8. Li N, peng Song Y, Tang H, Wang Y. Recent developments in sample preparation and data pre-treatment in metabonomics research. Arch Biochem Biophys. 2016;589:4-9.

9. Nordström A, Want E, Northen T, Lehtiö J, Siuzdak G. Multiple ionization mass spectrometry strategy used to reveal the complexity of metabolomics. Anal Chem. 2008;80:421-9.

10. Blennow K, de Leon MJ, Zetterberg H. Alzheimer's disease. Lancet. 2006;368:387-403.

11. Maccioni RB, Muñoz JP, Barbeito L. The molecular bases of Alzheimer's disease and other neurodegenerative disorders. Arch Med Res. 2001;32:367-81.

12. González-Domínguez R, García-Barrera T, Gómez-Ariza JL. Characterization of metal profiles in serum during the progression of Alzheimer's disease. Metallomics. 2014;9:292-300.

13. Ibáñez C, Simó C, Cifuentes A. Metabolomics in Alzheimer's disease research. Electrophoresis. 2013;34:2799-811.

14. Patel S, Shah RJ, Coleman P, Sabbagh M. Potential peripheral biomarkers for the diagnosis of Alzheimer's disease. Int J Alzheimers Dis. 2011;2011:572495.

15. González-Domínguez R, García-Barrera T, Gómez-Ariza JL. Metabolomic study of lipids in serum for biomarker discovery in Alzheimer's disease using direct infusion mass spectrometry. J Pharm Biomed Anal. 2014;98:321-6.

16. González-Domínguez R, García-Barrera T, Gómez-Ariza JL. Using direct infusion mass spectrometry for serum metabolomics in Alzheimer's disease. Anal Bioanal Chem. 2014;406:7137-48.

17. Beckmann M, Parker D, Enot DP, Duval E, Draper J. Highthroughput, nontargeted metabolite fingerprinting using nominal mass flow injection electrospray mass spectrometry. Nat Protoc. 2008;3:486-504.

18. Sumner LW, Amberg A, Barrett D, Beale MH, Beger R, Daykin CA, Fan TWM, Fiehn O, Goodacre R, Griffin JL, Hankemeier T, Hardy N, Harnly J, Higashi R, Kopka J, Lane AN, Lindon JC, Marriott P, Nicholls AW, Reily MD, Thaden JJ, Viant MR. Proposed minimum reporting standards for chemical analysis: chemical Analysis Working Group (CAWG) Metabolomics Standards Initiative (MSI). Metabolomics. 2007;3:211-21.

19. Vinaixa M, Schymanski EL, Neumann S, Navarro M, Salek RM, Yanes O. Mass spectral databases for LC/MS- and GC/MS-based metabolomics: state of the field and future prospects. Trends Anal Chem. 2016;78:23-35.

20. Psychogios N, Hau DD, Peng J, Guo AC, Mandal R, Bouatra S, Sinelnikov I, Krishnamurthy R, Eisner R, Gautam B, Young N, Xia J, Knox C, Dong E, Huang P, Hollander Z, Pedersen TL, Smith SR, Bamforth F, Greiner R, McManus B, Newman JW, Goodfriend T, Wishart DS. The human serum metabolome. PLoS ONE. 2011;6:e16957.

21. Moco S, Bino RJ, De Vos RCH, Vervoort J. Metabolomics technologies and metabolite identification. Trends Anal Chem. 2007;26:855-66.

22. Murphy RC, Barkley RM, Berry KZ, Hankin J, Harrison K, Johnson C, Krank J, McAnoy A, Uhlson C, Zarini S. Electrospray ionization and tandem mass spectrometry of eicosanoids. Anal Biochem. 2005;346:1-42. 
23. Vernez L, Hopfgartner G, Wenk M, Krahenbuhl S. Determination of carnitine and acylcarnitines in urine by high-performance liquid chromatography-electrospray ionization ion trap tandem mass spectrometry. J Chromatogr A. 2003;984:203-13.

24. Divito EB, Davic AP, Johnson ME, Cascio M. Electrospray ionization and collision induced dissociation mass spectrometry of primary fatty acid amides. Anal Chem. 2012;84:2388-94.

25. Murphy RC, Leiker TJ, Barkley RM. Glycerolipid and cholesterol ester analyses in biological samples by mass spectrometry. Biochim Biophys Acta. 2011;1811:776-83.

26. González-Domínguez R, García-Barrera T, Gómez-Ariza JL. Iberian ham typification by direct infusion electrospray and photospray ionization mass spectrometry fingerprinting. Rapid Commun Mass Spectrom. 2012;26:835-44.

27. Pulfer M, Murphy RC. Electrospray mass spectrometry of phospholipids. Mass Spectrom Rev. 2003;22:332-64.

28. Wang C, Xie S, Yang J, Yang Q, Xu G. Structural identification of human blood phospholipids using liquid chromatography/ quadrupole-linear ion trap mass spectrometry. Anal Chim Acta. 2004;525:1-10.

29. González-Domínguez R, García-Barrera T, Gómez-Ariza JL. Combination of metabolomic and phospholipid-profiling approaches for the study of Alzheimer's disease. J Proteom. 2014;104:37-47.

30. Haynes CA, Allegood JC, Park H, Sullards MC. Sphingolipidomics: methods for the comprehensive analysis of sphingolipids. J Chromatogr B. 2009;877:2696-708.

31. Liebisch G, Binder M, Schifferer R, Langmann T, Schulz B, Schmitz G. High throughput quantification of cholesterol and cholesteryl ester by electrospray ionization tandem mass spectrometry (ESI-MS/MS). Biochim Biophys Acta. 2006;1761:121-8.

32. Qiao X, Ye M, Liu CF, Yang WZ, Miao WJ, Dong J, Guo DA. A tandem mass spectrometric study of bile acids: interpretation of fragmentation pathways and differentiation of steroid isomers. Steroids. 2012;77:204-11.

33. González-Domínguez R, García-Barrera T, Gómez-Ariza JL. Metabolomic approach to Alzheimer's disease diagnosis based on mass spectrometry. Chem Pap. 2012;66:829-35.

34. González-Domínguez R, García-Barrera T, Vitorica J, GómezAriza JL. Application of metabolomics based on direct mass spectrometry analysis for the elucidation of altered metabolic pathways in serum from the APP/PS1 transgenic model of Alzheimer's disease. J Pharm Biomed Anal. 2015;107:378-85.

35. González-Domínguez R, García-Barrera T, Vitorica J, GómezAriza JL. Metabolomic research on the role of interleukin-4 in Alzheimer's disease. Metabolomics. 2015;11:1175-83.

36. González-Domínguez R, Rupérez FJ, García-Barrera T, Barbas C, Gómez-Ariza JL. Metabolomic-driven elucidation of pathological mechanisms associated with Alzheimer's disease and mild cognitive impairment. Curr Alzheimer Res. 2016;13:641-53.

37. González-Domínguez R, García-Barrera T, Vitorica J, GómezAriza JL. Deciphering metabolic abnormalities associated with Alzheimer's disease in the APP/PS1 mouse model using integrated metabolomic approaches. Biochimie. 2015;110:119-28.

38. González-Domínguez R, García-Barrera T, Gómez-Ariza JL. Metabolite profiling for the identification of altered metabolic pathways in Alzheimer's disease. J Pharm Biomed Anal. 2015;107:75-81.

39. González-Domínguez R, García A, García-Barrera T, Barbas C, Gómez-Ariza JL. Metabolomic profiling of serum in the progression of Alzheimer's disease by capillary electrophoresismass spectrometry. Electrophoresis. 2014;35:3321-30.

40. González-Domínguez R, Castilla-Quintero R, García-Barrera T, Gómez-Ariza JL. Development of a metabolomic approach based on urine samples and direct infusion mass spectrometry. Anal Biochem. 2014;465:20-7.

41. González-Domínguez R, García-Barrera T, Vitorica J, GómezAriza JL. Metabolomic screening of regional brain alterations in the APP/PS1 transgenic model of Alzheimer's disease by direct infusion mass spectrometry. $\mathbf{J}$ Pharm Biomed Anal. 2015;102:425-35.

42. González-Domínguez R, García-Barrera T, Vitorica J, GómezAriza JL. High throughput multi-organ metabolomics in the APP/ PS1 mouse model of Alzheimer's disease. Electrophoresis. 2015;36:2237-49.

43. Gago-Tinoco A, González-Domínguez R, García-Barrera T, Blasco-Moreno J, Bebianno MJ, Gómez-Ariza JL. Metabolic signatures associated with environmental pollution by metals in Doñana National Park using P. clarkii as bioindicator. Environ Sci Pollut Res Int. 2014;21:13315-23.

44. Lin S, Liu H, Kanawati B, Liu L, Dong J, Li M, Huang J, Schmitt-Kopplin P, Cai Z. Hippocampal metabolomics using ultrahigh-resolution mass spectrometry reveals neuroinflammation from Alzheimer's disease in CRND8 mice. Anal Bioanal Chem. 2013;405:5105-17.

45. Lin S, Kanawati B, Liu L, Witting M, Li M, Huang J, SchmittKopplin P, Cai Z. Ultrahigh resolution mass spectrometry-based metabolic characterization reveals cerebellum as a disturbed region in two animal models. Talanta. 2014;118:45-53.

46. González-Domínguez R, García-Barrera T, Gómez-Ariza JL. Application of a novel metabolomics approach based on atmospheric pressure photoionization mass spectrometry using flow injection analysis for the study of Alzheimer's disease. Talanta. 2015;131:480-9.

47. Wood PL, Locke VA, Herling P, Passaro A, Vigna GB, Volpato S, Valacchi G, Cervellati C, Zuliani G. Targeted lipidomics distinguishes patient subgroups in mild cognitive impairment (MCI) and late onset Alzheimer's disease (LOAD). BBA Clin. 2016;5:25-8

48. Han X, Holtzman DM, McKeel DW Jr. Plasmalogen deficiency in early Alzheimer's disease subjects and in animal models: molecular characterization using electrospray ionization mass spectrometry. J Neurochem. 2001;77:1168-80.

49. Han X, Holtzman DM, McKeel DW Jr, Kelley J, Morris JC. Substantial sulfatide deficiency and ceramide elevation in very early Alzheimer's disease: potential role in disease pathogenesis. J Neurochem. 2002;82:809-18.

50. Cheng H, Zhou Y, Holtzman DM, Han X. Apolipoprotein E mediates sulfatide depletion in animal models of Alzheimer's disease. Neurobiol Aging. 2010;31:1188-96.

51. Cheng H, Wang M, Li JL, Cairns NJ, Han X. Specific changes of sulfatide levels in individuals with pre-clinical Alzheimer's disease: an early event in disease pathogenesis. J Neurochem. 2013;127:733-8.

52. Han X, Rozen S, Boyle SH, Hellegers C, Cheng H, Burke JR, Welsh-Bohmer KA, Doraiswamy PM, Kaddurah-Daouk R. Metabolomics in early Alzheimer's disease: identification of altered plasma sphingolipidome using shotgun lipidomics. PLoS ONE. 2011;6:e21643. 